\title{
Src kinase up-regulates the ERK cascade through inactivation of protein phosphatase 2A following cerebral ischemia
} Xiaohan $\mathrm{Hu}^{\dagger 1,2}$, Xiangyang $\mathrm{Wu}^{\dagger 1,2,3}$, Jiali $\mathrm{Xu}^{2,4}$, Jin $\mathrm{Zhou}^{2,3}$, Xiao Han ${ }^{1,2}$ and Jun Guo*1,2,4

Address: ${ }^{1}$ Key Laboratory of Human Functional Genomics of Jiangsu Province, Nanjing Medical University, Nanjing 210029, PR China, 2Department of Biochemistry, Nanjing Medical University, Nanjing 210029, PR China, ${ }^{3}$ Department of General Surgery, The Nanjing First Hospital Affiliated to Nanjing Medical University, Nanjing 210006, PR China and ${ }^{4}$ Laboratory Center for Basic Medical Sciences, Nanjing medical university Nanjing, 210029, PR China

Email: Xiaohan Hu - Xiaoada@gmail.com; Xiangyang Wu - Wuhww@yahoo.com.cn; Jiali Xu - Scarlett0830@126.com; Jin Zhou - zhoujing6@sina.com; Xiao Han - hanxiao@njmu.edu.cn; Jun Guo* - Guoj69@yahoo.com.cn

* Corresponding author †Equal contributors

Published: 14 July 2009

BMC Neuroscience 2009, 10:74 doi:10.1 186/1471-2202-10-74
Received: 25 March 2009

Accepted: 14 July 2009

This article is available from: http://www.biomedcentral.com/I47/-2202//0/74

(c) $2009 \mathrm{Hu}$ et al; licensee BioMed Central Ltd.

This is an Open Access article distributed under the terms of the Creative Commons Attribution License (http://creativecommons.org/licenses/by/2.0), which permits unrestricted use, distribution, and reproduction in any medium, provided the original work is properly cited.

\begin{abstract}
Background: The regulation of protein phosphorylation requires a balance in the activity of protein kinases and protein phosphatases. Our previous data indicates that Src can increase ERK activity through Raf kinase in response to ischemic stimuli. This study examined the molecular mechanisms by which Src activates ERK cascade through protein phosphatases following cerebral ischemia.

Results: Ischemia-induced Src activation is followed by phosphorylation of PP2A at Tyr307 leading to its inhibition in the rat hippocampus. SU6656, a Src inhibitor, up-regulates PP2A activity, resulting in a significant decreased activity in ERK and its targets, CREB and ER $\alpha$. In addition, the PP2A inhibitor, cantharidin, led to an up-regulation of ERK activity and was able to counteract Src inhibition during ischemia.

Conclusion: Src induces up-regulation of ERK activity and its target transcription factors, CREB and ER $\alpha$, through attenuation of PP2A activity. Therefore, activation of ERK is the result of a crosstalk between two pathways, Raf-dependent positive regulators and PP2A-dependent negative regulators.
\end{abstract}

\section{Background}

When blood flow is decreased to the brain, cerebral ischemia, a complex signaling network is activated. Excitotoxicity can be induced by an increase in intraneuronal $\mathrm{Ca}^{2+}$ through calcium ion channels, such as the N-methylD-aspartic acid (NMDA) receptor, L-type voltage-gated calcium channels (L-VGCC) and IP3 receptor. An influx in intraneuronal $\mathrm{Ca}^{2+}$ is a key mediator in multiple intracellular signaling cascades after ischemia [1]. Extracellular signal - regulated kinase (ERK), a member of the mitogen- activated protein kinase (MAPK) family, is activated in a $\mathrm{Ca}^{2+}$-dependent manner in cerebral ischemia [2-4]. ERK is regulated via phosphorylation at various motifs. Previous studies have demonstrated that ERK is activated by various upstream kinases, such as Akt and Src, by the Raf/ MEK/ERK signaling cascade [5-7]. In response to ischemic stimuli, active Src kinase activates ERK through Raf phosphorylation at Tyr340/Tyr341 [8]. Src is a non-receptor protein tyrosine kinase (PTK). The Src family of proteins contain a Src homology (SH) 2 domain and SH3 domain, 
a catalytic domain, and a C-terminal tail. Src maintains basal activity in normal cells by an auto-inhibitory mechanism, whereby Tyr527 in the C-terminal tail is phosphorylated allowing for association with the $\mathrm{SH} 2$ domain preventing catalytic activity. Src activation is initiated when Tyr527 is dephosphorylated and, subsequently, autophosphorylated at Tyr-416 [7].

In general, changes in protein phosphorylation require coordinate regulation of protein kinases and protein phosphatases. However, to date, the signaling mechanisms leading to dephosphorylation of ERK resulting in inactivation have not been well defined. Protein phosphotase 2A (PP2A) has been shown to be an ERK phosphatase. Furthermore, it has been suggested that PP2A dephosphorylates critical residues resulting in ERK inactivation [9-11]. PP2A is a Ser/Thr-specific phosphatase composed of two regulatory (A and $\mathrm{B}$ ) and one catalytic subunit (C) [12]. The catalytic subunit of PP2A (PP2AC) is regulated by phosphorylation at Tyr307 in the con- served C-terminal domain of the catalytic subunit resulting in inactivation of the enzyme $[12,13]$. Active Src can directly phosphorylate PP2A C at Tyr307 [14] and cerebral ischemia leads to up-regulation of Src activity [15]. However, whether Src kinase induces ERK activation through inhibition of PP2A during cerebral ischemia is unclear.

Following ischemia, the activated ERK cascade regulates gene expression via upregulation of specific transcription factors [16]. One of the upregulated targets is estrogen receptor $\alpha(E R \alpha)$, a neuroprotector. It has been reported that ER $\alpha$ phosphorylation at Ser118 positively regulates its function and that phospho-ERK phosphorylates this residue [17-20]. Another neuroprotective protein induced by ERK activation is cyclic AMP response element-binding protein (CREB). Additionally, ERK phosphorylates CREB at Ser133, which is essential for CREB-mediated effects on transcription [21,22]. Interestingly, this residue has been shown to be dephosphorylated by PP2A [23,24].

A

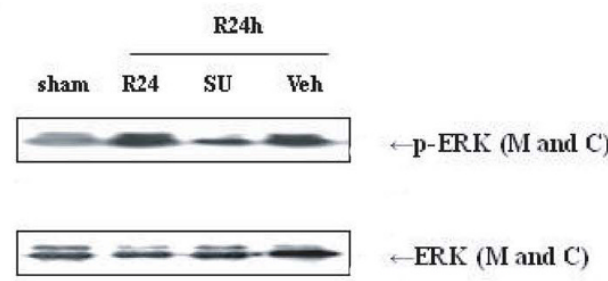

B
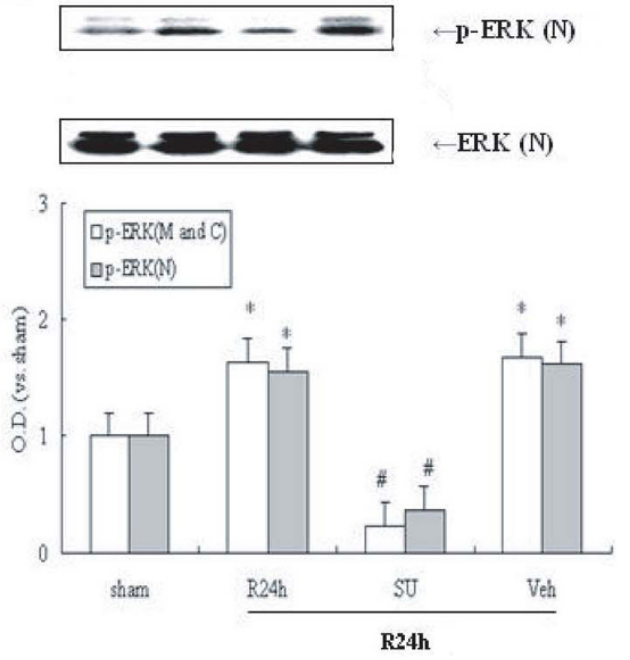

$\mathrm{C}$

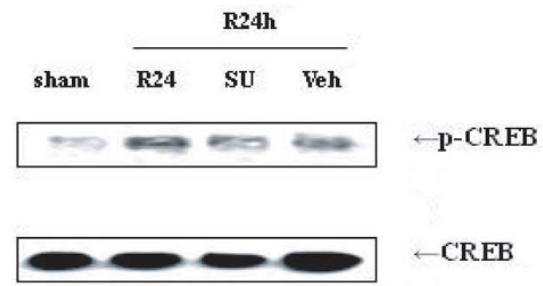

D
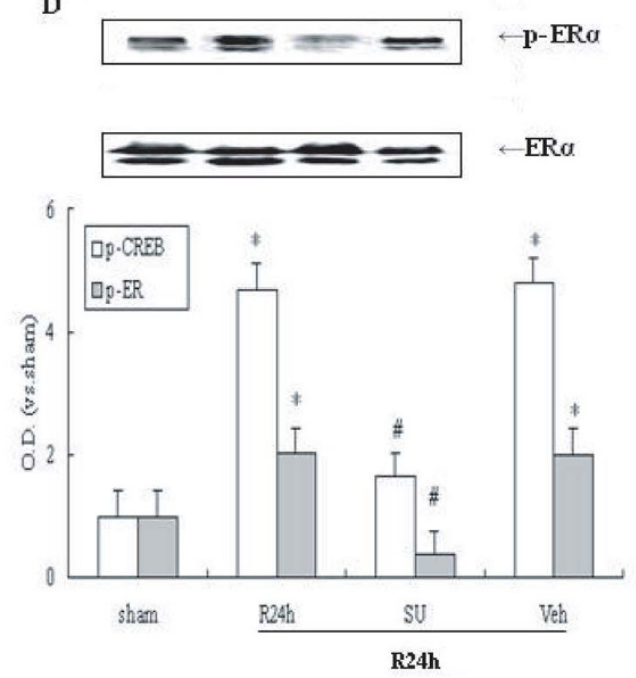

Figure I

Western blot analyses of p-ERK, p-CREB and p-ER $\alpha$ protein levels and the effect of Src inhibition on them following cerebral ischemia. SU6656 (SU) and its vehicle (Veh) were administered 20 min before ischemia (I.c.v.) respectively. $24 \mathrm{~h}$ reperfusion after $10 \mathrm{~min}$ ischemia was performed. (A) Effect of SU on P-ERK (Thr202/Tyr204) in plasma membrane and cytoplasm ( $M$ and $C$ ) following cerebral ischemia, (B) Effect of SU on p-ERK in nucleus (N) post-ischemia, (C) Role of SU in pCREB (Ser I I8) following post-ischemic reperfusion, (D) Role of SU in P-ER $\alpha$ (Ser I 33) during reperfusion post-ischemia. Bands were scanned and the optical density (O.D.) is presented as fold-increase compared to sham control levels. Data are expressed as mean \pm S.D. $(n=4 /$ group), $\# P<0.05$ vs. the respective control group. 
Therefore, in the present study, we examine the involvement of PP2A in Src-dependent ERK phosphorylation in the rat hippocampus following ischemia. Through the use of SU6656 (SU), a Src inhibitor, we show that PP2A activity is upregulated, which may, in turn, attenuate ERK activation and its downstream proteins, CREB and ER $\alpha$, in the post-ischemic hippocampus. Thus, we propose that Src induces ERK activation through downregulation of PP2A activity in cerebral ischemia.

\section{Results}

Src inhibitor decreases activity of the ERKICREB and ER $\alpha$ pathways post-ischemia

ERK can be expressed widely and is found in the cell membrane, cytoplasm, and nucleus [16]. Some previous studies have suggested that the ERK signaling cascade may be up-regulated in a Src-dependent manner following cerebral ischemia $[15,25]$. Therefore, it was determined if Src kinase regulates ERK activity at different and specific sub- cellular sites in response to ischemic stimuli. ERK and pERK were measured by immunoblot in defined subcellular regions, including the cell membrane, cytoplasm and nucleus. SU6656 (SU, a selective inhibitor of Src) was used to inhibit Src activity. Rats underwent 4-VO and endured $10 \mathrm{~min}$ ischemia followed by $24 \mathrm{~h}$ reperfusion. Ischemia leads to phosphorylation of ERK during the 24 $h$ reperfusion post-ischemia in the plasma membrane and cytoplasm (M and C) and nucleus (N) (Figure. 1A and 1B, $P<0.05)$. SU effectively attenuated ERK phosphorylation after $24 \mathrm{~h}$ reperfusion. In addition, no changes were observed in the total protein levels of ERK. These data indicate that cerebral ischemia results in an increase in ERK activity which is independent of subcellular localization, but dependent on Src activation in the post-ischemic hippocampus.

Activated ERK can facilitate the phosphorylation of a variety of transcription factors phosphorylation involved in
A

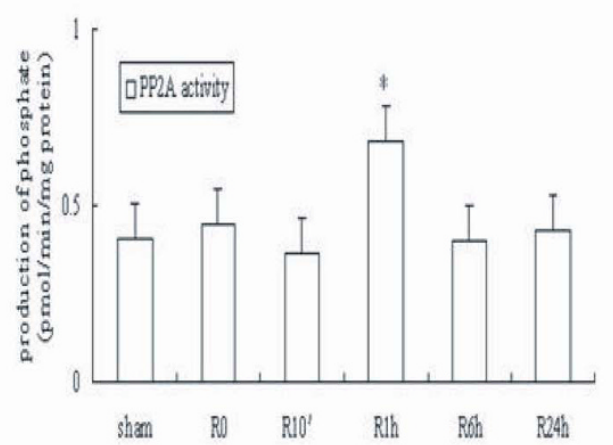

B

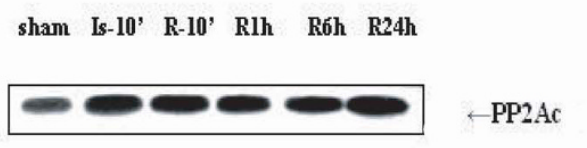

C

sham Is-10' R-10' Rlh R6h R24h

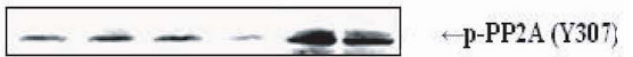

D
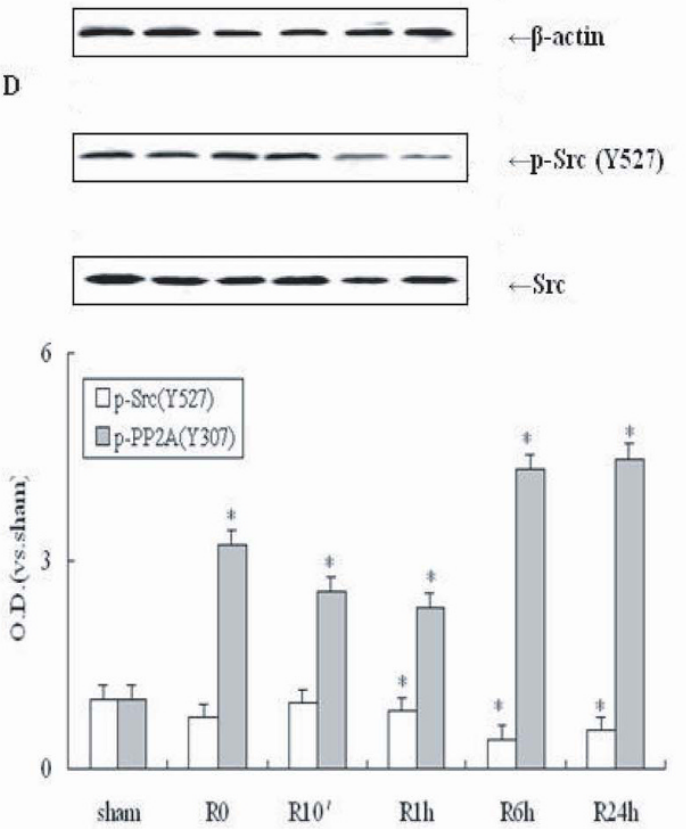

Figure 2

Western blot analyses of p-Src and p-PP2A C levels following post-ischemic reperfusion. Samples were taken from the hippocampi of rats subjected to sham, or $0,10 \mathrm{~min}, \mathrm{I} \mathrm{h}, 6 \mathrm{~h}$ and $24 \mathrm{~h}$ reperfusion after 10 min ischemia. PP2A activity was measured using a molybdate dye-based phosphatase assay kit. Sample proteins were identified by special antibodies against $P-$ Src (Tyr527), Src, PP2A C and P-PP2A C (Tyr307). (A) Temporal curve of PP2A activity following post-ischemic reperfusion. (B) Temporal alterations of PP2A C levels during reperfusion post-ischemia. (C) Temporal alterations of $P$-PP2A C and $\beta$-actin levels following post-ischemic reperfusion. (D) Temporal alterations of $p$-Src and Src protein levels after post-ischemic reperfusion. O.D. is presented as fold-increase compared to sham control levels. Data are expressed as mean \pm S.D. ( $n=4 / g r o u p)$, $* P<0.05$ vs. sham control. 
A
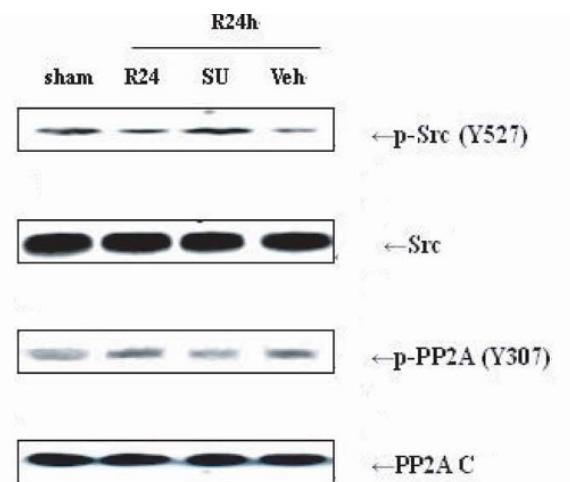

C
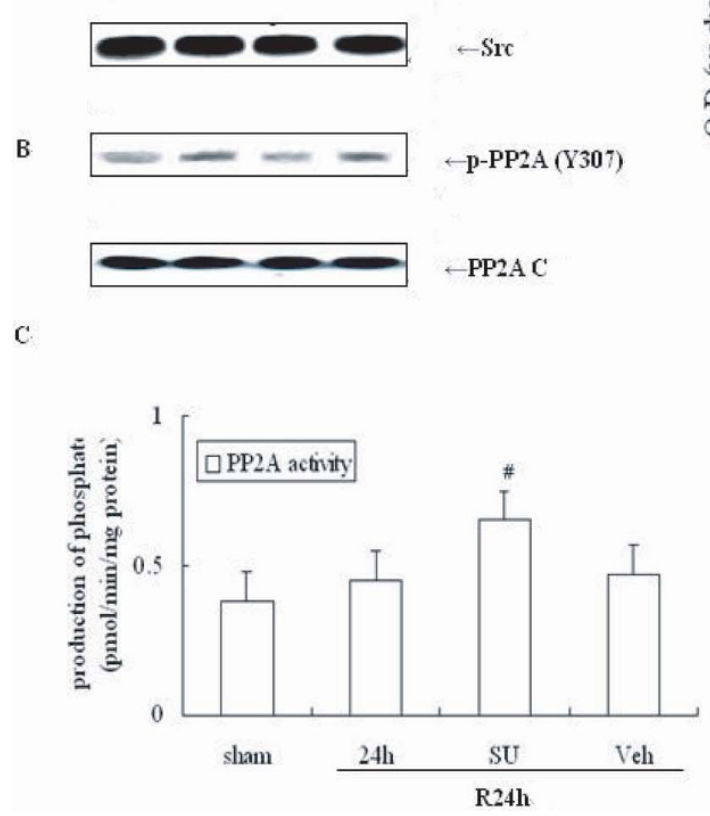

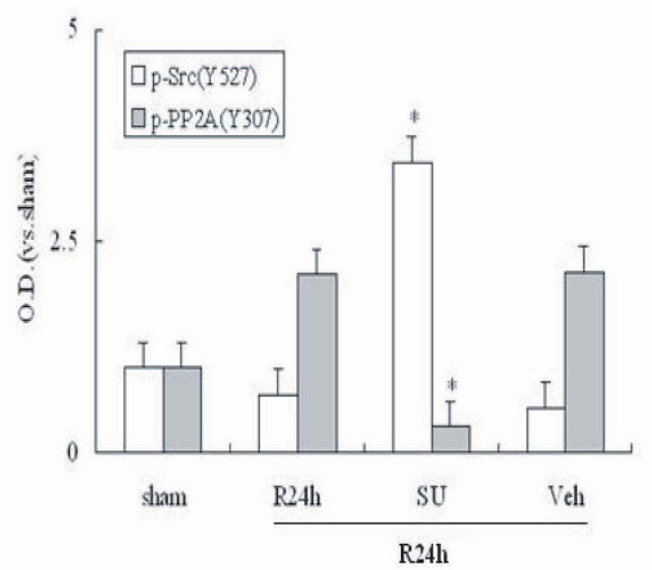

D

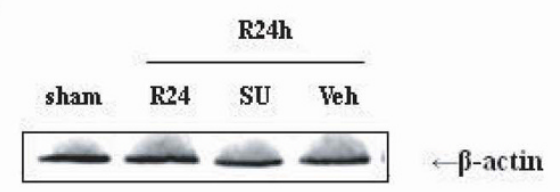

\section{Figure 3}

Effects of SU on the Src/PP2A C signaling pathways in response to cerebral ischemia. SU or its Veh was administered into the left intracerebral ventricle $20 \mathrm{~min}$ prior to occlusion. $24 \mathrm{~h}$ post-ischemic reperfusion was performed. Proteins were measured using the same antibodies mentioned in Figure 2. (A) Determination of $p$-Src levels with and without SU during $24 \mathrm{~h}$ reperfusion following $10 \mathrm{~min}$ ischemia. (B) Determination of P-PP2A C levels with and without SU following cerebral ischemia. (C) Alteration of PP2A C activity following post-ischemic reperfusion. (D) $\beta$-actin with and without SU during reperfusion post-ischemia. O.D. is presented as fold-increase compared to sham control levels. Data are expressed as mean \pm S.D. ( $n=4 /$ group), $* P<0.05$ vs. sham control.

gene expression [16]. Two proteins regulated by ERK are CREB and $E R \alpha$, which are activated by phosphorylation at the Ser133 residue [22] and at the Ser118 site [20], respectively. In this study, CREB and ER $\alpha$ nuclear activity were observed in response to cerebral ischemia. Compared with a sham group, both phospho-CREB and phospho$\mathrm{ER} \alpha$ are increased in the $24 \mathrm{~h}$ reperfusion groups (Figure. $1 \mathrm{C}$ and $1 \mathrm{D} ; P<0.05)$ and similar to ischemia-induced ERK activity. To determine whether Src might regulate CREB and ER $\alpha$ activity following ischemia, SU was employed. Levels of p-ER $\alpha$ and p-CREB in the $24 \mathrm{~h}$ reperfusion group showed obvious decrease in animals in which SU was administered (Figure. $1 \mathrm{C}$ and $1 \mathrm{D} ; \mathrm{P}<0.05$ ). These data suggest that Src kinase is required for activation of ERK and, subsequently, ER $\alpha$ and CREB post-ischemic hippocampus.

\section{Src activation is correlated with an increase in PP2A} phosphorylation and inhibition

In general, ERK, ER $\alpha$ and CREB phosphorylation are determined by a balance in the activity of upstream kinases and phosphatases. It has been suggested that the Ser/Thr-specific phosphatase, PP2A, might negatively regulate ERK, ER $\alpha$ and CREB activity [26-28]. To examine whether PP2A is involved in the regulation of the Src/ERK pathway post-ischemia, it was first assessed whether ischemia-induced alteration of PP2A activity. All samples were from rats subjected to various reperfusion times $(0$, $10 \mathrm{~min}, 1 \mathrm{~h}, 6 \mathrm{~h}$ and $24 \mathrm{~h}$ ) after $10 \mathrm{~min}$ ischemia. Tissue extracts of the hippocampi were processed and assayed using a PP2A activity assay system. The peak of PP2A activity was observed at approximately $1 \mathrm{~h}$ of reperfusion (approximately 1.5 times higher than the sham group, Figure. $2 \mathrm{~A}, P<0.05)$. Sustained inactivation of PP2A activity was observed after $6 \mathrm{~h}$ and $24 \mathrm{~h}$ of reperfusion and was concomitant with upregulation of the ERK cascade. In addition, no changes were observed in the total protein of PP2A C (Figure. 2B, $P>0.05$ ). To confirm inhibition of PP2A activity, immunoblot were performed to assess PP2A phosphorylation at the Tyr307 site in the hippocampus during post-ischemic reperfusion. Hippocampal tissue extracts were prepared as previously described for 
Figure. 1. As shown in Figure. 2C, ischemia resulted in marked dephosphorylation of PP2A at Tyr307 after $1 \mathrm{~h}$ reperfusion, indicating that $\mathrm{PP} 2 \mathrm{~A}$ activation was induced by ischemia. However, significant phosphorylation of PP2A at Tyr307 was observed after 6 h reperfusion $(P<$ $0.05)$, indicating sustained inactivation of PP2A. Active Src kinase directly phosphorylates PP2A at Tyr307 $[12,29]$. Therefore, it was determined whether Src is required for inactivation of PP2A in cerebral ischemia. Induction of cerebral ischemia results in dephosphorylation of Src at Tyr527 increasing its activity at by $6 \mathrm{~h}$ reperfusion (Figure. 2D, $P<0.05$ ). Therefore, ischemiainduced Src activation is accompanied by PP2A inhibition. No changes were observed in the total protein of Src and PP2A in each group (Figure $2 \mathrm{~B}-\mathrm{D}, P>0.05$ ). $\beta$-actin protein levels, used as a control, also remained stable in each group (Figure 2C, $P>0.05$ ).

A
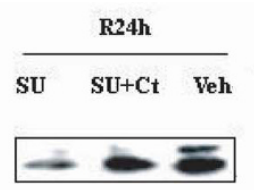

$\leftarrow$-ERK (M and C)

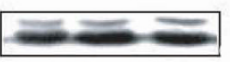

$\leftarrow$ ERK (M and C)

B
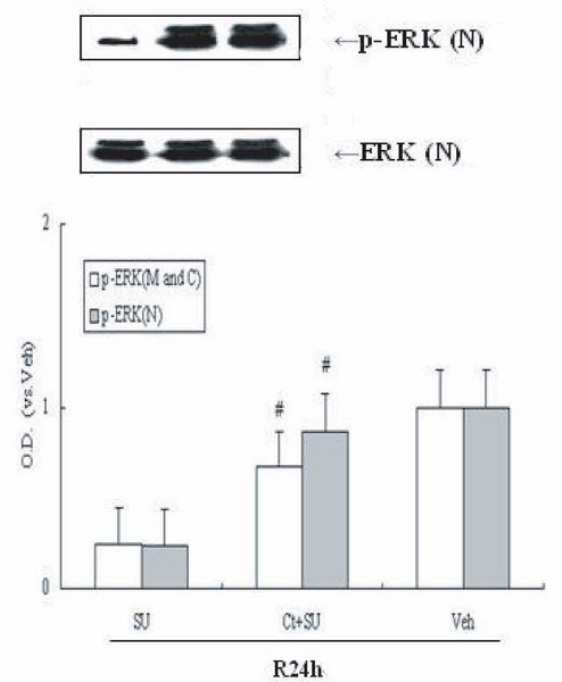

Inhibition of Src activity results in PP2A increased activity in response to cerebral ischemia

The results presented above suggest that activated Src kinase likely regulates PP2A activity through phosphorylation at Tyr307 following cerebral ischemia. In order to determine whether Src activation is required for inactivation of PP2A in cerebral ischemia SU was employed. Immunoblot was performed to assess p-Src and p-PP2A levels in the hippocampi of ischemic animals. Rats underwent $4-\mathrm{VO}$ and endured 10-min ischemia followed by 24 $\mathrm{h}$ reperfusion. As shown in Figure 3A, SU produced an additive increase in Src phosphorylation at Tyr527 site resulting in a decrease in Src activity, since Tyr527 is an inhibitive site of Src. In contrast, SU also inhibited PP2A phosphorylation at Tyr307 after $24 \mathrm{~h}$ reperfusion (Figure $3 \mathrm{~B}, P<0.05)$. These data indicate that Src activation is required for PP2A phosphorylation following ischemia. These same tissue extracts were processed and assayed

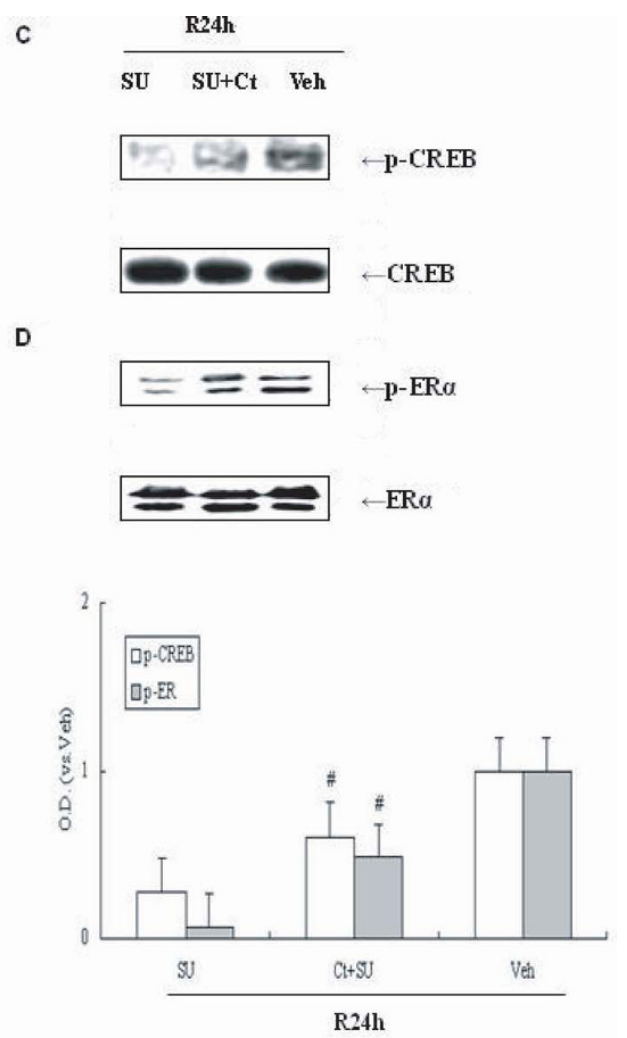

\section{Figure 4}

Western blot analyses of p-ERK, p-CREB and p-ER $\alpha$ protein levels and the effects of Ct and (or) SU on them in cerebral ischemia. Cantharidin $(\mathrm{Ct})$ and (or) SU were administered at $24 \mathrm{~h}$ reperfusion after ischemia. (A) Effect of SU and co-administration with $C_{t}$ and $S U$ on $\mathrm{P}$-ERK in plasma membrane and cytoplasm ( $M$ and $\left.C\right)$ following post-ischemic reperfusion, (B) Effect of SU and co-administration with Ct and SU on P - ERK in nucleus (N), (C) Effect of SU and co-administration with $\mathrm{Ct}$ and $\mathrm{SU}$ on $\mathrm{p}-\mathrm{CREB}$ following post-ischemic reperfusion, (D) Effect of SU and co-administration with $\mathrm{Ct}$ on $\mathrm{P}-\mathrm{ER} \alpha$ following post-ischemic reperfusion. The results are expressed as fold changes versus the sham controls. O.D. is presented as fold - increase compared to Veh controls. Data are expressed as mean \pm S.D. ( $n=4$ /group), \#P< 0.05 vs. the respective SU administration group. 


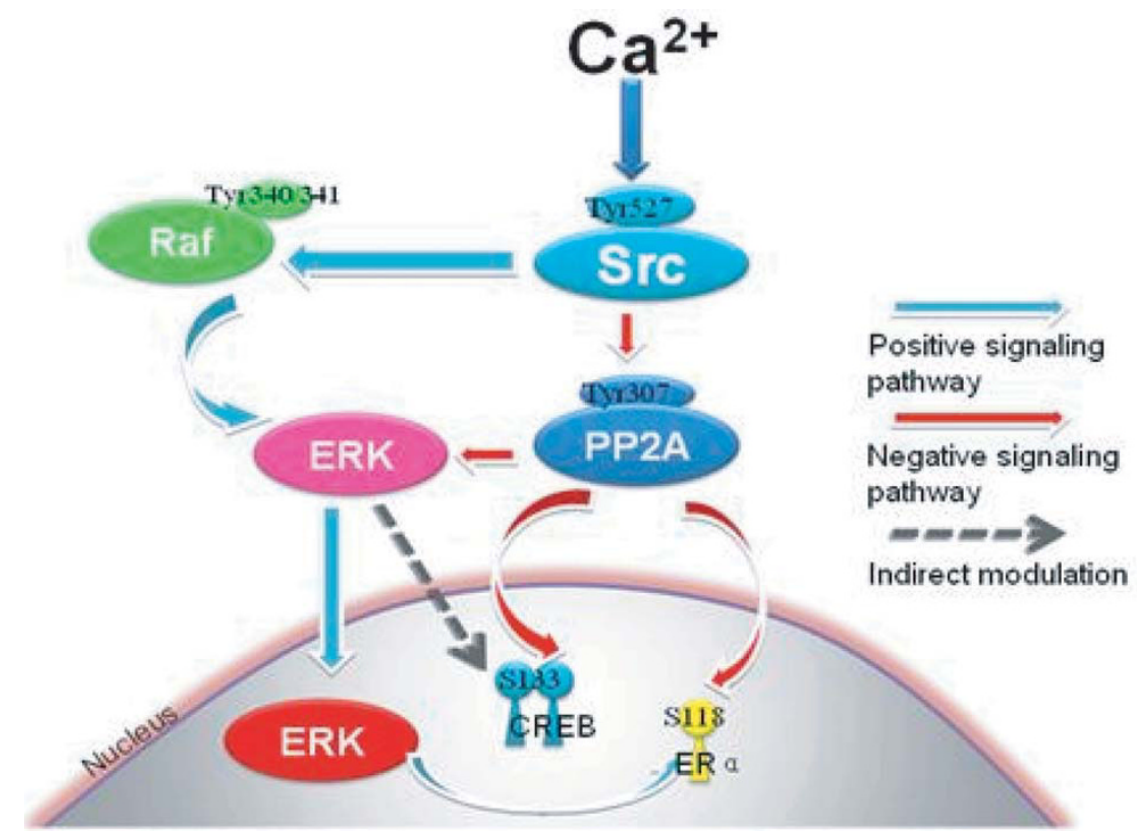

\section{Figure 5}

Crosstalk between Raf-dependent, positively regulated signal cascades and a PP2A-dependent negative signaling pathway in cerebral ischemia and reperfusion. In response to ischemia-induced increases in intracellular $\mathrm{Ca}^{2+}$, active Src kinase up-regulated ERK cascades through increment of Raf-I phosphorylation at its active site (Tyr340/34I). Meanwhile, Src kinase also contributes to ERK activation through increment of PP2A phosphorylation at its inactivation site (Tyr307) in cerebral ischemia. These two converse signaling pathways undergo crosstalk through ERK and play important roles in ERK/ CREB and ER $\alpha$ cascades following cerebral ischemia.

using a PP2A activity assay system. As expected, the PP2A activity was higher in samples treated with SU compared with the sham group (Figure $3 \mathrm{C}, P<0.05$ ). Furthermore, the total protein levels of Src, PP2A $\mathrm{c}$ and $\beta$-actin remained unchanged in each group (Figure $3 \mathrm{~A}-3 \mathrm{D}, P>$ $0.05)$. These data show that Src activation is required for PP2A inhibition following cerebral ischemia.

\section{Inhibition of PP2A compensates for inhibition of Src allowing for upregulation of ERKICREB and ER $\alpha$ in the presence of SU}

Although activated Src kinase decreases PP2A activity after cerebral ischemia, it is not known whether PP2A is involved in the Src/ERK cascade following cerebral ischemia. SU and Cantharidin (Ct), a PP2A inhibitor, was administered prior to ischemia (i.c.v.), and ERK and pERK protein were examined in the cell membrane, cytoplasm and nucleus of post-ischemic hippocampi. As shown in Figure 4A, samples from animals treated with both Ct and SU had significantly higher ERK phosphorylation compared with those samples treated with SU only $(P<0.05)$. These data demonstrate that ischemia induces Src activation leading to inhibition of PP2A activity resulting in ERK activation.

PP2A is also able to dephosphorylate ERK targets, CREB and ER $\alpha$. To further assess the role of PP2A in regulation of signaling cascades during cerebral ischemia, intranuclear CREB and ER $\alpha$ immunoblot assays were performed using specific phosphorylation antibodies. Compared to samples treated with SU only, both ER $\alpha$ and CREB phosphorylations are increased in the $\mathrm{Ct}$ and SU co-administration samples $(\mathrm{P}<0.05$; Figure $1 \mathrm{~B})$. These data suggest that Src is required for up-regulation of CREB and ER $\alpha$ pathway through inhibition of PP2A activity. Total protein of ERK, CREB and ER $\alpha$ in each group remained unchanged (Figure 4A-B, $P>0.05$ ).

\section{Discussion}

The Raf/ERK pathway couples receptor tyrosine kinase (RTK) to cell fate decisions, such as growth, proliferation, migration, differentiation and survival [30]. It is well known that non-receptor tyrosine kinases, such as Src, can activate the ERK cascade [6,7]. Our previous studies have indicated that Src kinase can up-regulate the ERK cascade through direct phosphorylation of Raf at Tyr340/Tyr341 in response to ischemic stroke [15]. Here, a novel mechanism was identified whereby Src kinase induces the ERK pathway in a PP2A-dependent manner in rat hippocampus following ischemia (Figure 5).

PP2A is a Ser/Thr-specific phosphatase capable of dephosphorylating and inactivating ERK $[9,10,29]$. Induction of Src results in inactivation of PP2A resulting in up-regula- 
tion of ERK activity in cerebral ischemia. Several lines of evidence support the role of PP2A in regulation of the Src/ ERK pathway. First, cerebral ischemia results in sustained activation of Src kinase after $6 \mathrm{~h}$ reperfusion postischemia, accompanied by continuous phosphorylation of Tyr307 and inhibition of PP2A. Second, SU6656, an effective Src inhibitor, prevents PP2A phosphorylation resulting in up-regulation of PP2A activity. Third, cantharidin is a specific inhibitor of PP2A, which has little effect on PP1. Treatment with cantharidin abrogates the effects of the Src inhibitor, SU6656, allowing for upregulation of ERK activity following ischemia. These results indicate that Src upregulation of the ERK pathway in ischemic neurons requires inhibition of PP2A.

Src-induced phosphorylation and inactivation of PP2A was thought to be closely associated with intracellular calcium signaling [12]. In rat hippocampal neurons, the Src/ ERK cascade is dependent on calcium influx elicited by upregulation of ion channels like NMDA receptor and IP3 receptor. In addition, inhibition of ion channels can inhibit Src and ERK activity after cerebral ischemia $[2,4,31]$. Our previous studies have also suggested that Src kinase can up-regulate the Raf/ERK cascade directly in a calcium-dependent manner following ischemia stroke [6]. Apparently, Src can activate the ERK cascade via coordinated activation of protein kinases and inactivation of protein phosphatases in a calcium-dependent manner.

ERK exert their function through up-regulation of nuclear transcription factors resulting in changes in gene expression [16]. Our present study indicates that there were no changes in subcellular localization of total protein levels of ERK in response to ischemic stimuli. Cerebral ischemia induced an increase in ERK phosphorylation and activity in membrane, cytoplasma, and nucleus in hippocampal neurons. Activated ERK in the nucleus is sufficient to target its intranuclear substrates like CREB and ERo. As transcription factors, CREB and ER $\alpha$ are localized primarily in the nucleus of rat hippocampal neurons and their activities are negatively regulated by PP2A. Therefore, CREB and ER $\alpha$ share similar mechanisms as downstream molecules of ERK, and are modulated by Src kinase through a complex signalling network dependent on PP2A inactivation. CREB and ER $\alpha$ are thought to be protective factors in the nucleus $[20,21]$. Upregulation of intranuclear ERK/ CREB and ER $\alpha$ pathway is involved in neuroprotective gene expression in response to Src signals following cerebral ischemia [32].

\section{Conclusion}

Activation of Src kinase in neurons during cerebral ischemia induces a complex signaling network leading to activation of ERK. In response to ischemia-induced upregulation of Src kinase, Raf, a positive regulator of the ERK signaling cascade, is activated. In addition induction of
Src kinase activity also leads to inhibition of PP2A, a negative regulator of ERK activity. Activation of ERK through induction of Raf activity and inhibition of PP2A leads to activation of CREB and ER $\alpha$, transcription factors, which is involved in neuronal protection from ischemic stress.

\section{Methods \\ Transient cerebral ischemia}

Adult male Sprague-Dawley rats weighing approximately $250 \mathrm{~g}$ (Shanghai Laboratory Animal Center, SLAC, China) were housed under natural conditions (12-h light/12-h dark cycle from 07:00 to 18:00 h) at a constant temperature of $25^{\circ} \mathrm{C}$ with food and water ad libitum. All animal surgery was performed in accordance with the Institutional Animal Care and Use Committee and conformed to international guidelines on the ethical use of animals (Guide for the Care and Use of Laboratory Animal, 1996). Before surgery, the animals were deprived of food and water overnight. Experimental rats were anesthetized with $20 \%$ chloral hydrate $(300 \mathrm{mg} / \mathrm{kg}$, i.p.) and subjected to four-vessel occlusion (4VO) according to the method of Pulsinelli et al., 1982. Briefly, in sterile conditions while animals were under deep anaesthesia, the bilateral vertebral arteries were occluded permanently by electrocauterization and the common carotid arteries were separated from connective tissue and nerves and marked with surgical thread. On the following day, ischemia was induced by bilateral occlusion of the common carotid arteries for 10 min using aneurysm clips. To minimize the experimental variations, all rats used had to satisfy the following criteria: (1) complete flat electroencephalogram throughout the duration of carotid occlusion, (2) maintenance of dilated pupils and an absence of cornea reflex during ischemia, (3) rigor of the extremities and vertebral column. Sham operated control rats received the same treatment without carotid artery occlusion. Body temperature was monitored using a rectal probe and maintained at approximately $37^{\circ} \mathrm{C}$ using a heating pad until the animal had fully recovered from anesthesia.

\section{I.c.v. infusion and administration of inhibitors}

Ten microliter microsyringes were used for injections. Polyethylene tubing was used to attach injection cannula to the microsyringe. A burr hole was drilled in the skull measured from bregma $(0.8 \mathrm{~mm}$ posterior, $1.5 \mathrm{~mm}$ lateral and $3.5 \mathrm{~mm}$ deep) for administration of chemicals or vehicle before ischemia. Three microliters of cantharidin $(\mathrm{Ct}, 25 \mu \mathrm{g} / \mu \mathrm{l}$; Biomol, Plymouth Meeting, PA) and SU6656 (SU, $5 \mu \mathrm{M}$; Calbiochem, La Jolla, CA, USA) (in DMSO) were administered, either individually or in combination, into the cerebral ventricle (i.c.v.). After the injection, the injector was retained in place for an additional 5 min in order to reduce any possible backflow of the liquid along with the injection void. Occlusion occurred $20 \mathrm{~min}$ post-injection. 


\section{Subcellular fractionation and sample preparation}

Rats were euthanized by decapitation at various time points: $10 \mathrm{~min}$ after ischemia or $10 \mathrm{~min}, 1 \mathrm{~h}, 6 \mathrm{~h}$, or $24 \mathrm{~h}$ post-reperfusion. The hippocampi were quickly removed on ice in a cold room [6,33] and the separated brain regions were homogenized in 1:10 (w/v) ice-cold homogenization buffer A (HEPES 50, pH 7.4, KCl 100, $\mathrm{Na}_{3} \mathrm{VO}_{4}$ 1, NaF 50, and PMSF $1 \mathrm{mM}$ ) supplemented with $1 \%$ mammalian protease inhibitor cocktail (Sigma-Aldrich Co., St. Louis, MO). Cytoplasmic and membrane proteins were extracted by centrifugation at $800 \times g$ for $20 \mathrm{~min}$ at $4{ }^{\circ} \mathrm{C}$. Following centrifugation, the supernatant was transferred into the fresh tubes, which containing the membrane and cytoplasmic proteins ( $\mathrm{M}$ and $\mathrm{C}$ ). The resulting pellet was resuspended in homogenization buffer $B$ (buffer A, 1 mM DTT, 1\% cocktail and 10\% NP-40), kept on ice for $30 \mathrm{~min}$, intensely shaken for $15 \mathrm{~min}$, and then centrifuged at $14,000 \times \mathrm{g}$ for $20 \mathrm{~min}$ at $4^{\circ} \mathrm{C}$. The supernatant contains the nuclear protein $(\mathrm{N})$. All of the supernatant was extracted and then stored at $-80^{\circ} \mathrm{C}$ until assayed. The protein concentrations of the extracts were determined according to the Bradford assay protocol using bovine serum albumin (BSA) as a standard (Bradford, 1976).

\section{Western blot analysis}

Equivalent amounts of protein lysates were resolved by $10 \%$ SDS-PAGE, transferred to nitrocellulose membrane, incubated in a solution of 3\% BSA-Block/TBST for $2 \mathrm{~h}$ at room temperature, and then incubated with primary antibody in $1 \%$ BSA with shaking at $4{ }^{\circ} \mathrm{C}$ overnight. Following three 5-10 min washes in TBST, the membranes were incubated with secondary antibody conjugated to horseradish peroxidase in 1\% BSA at room temperature for $2 \mathrm{~h}$. Following the incubation, membranes were given three 10-min washes with TBST, and then developed using the Pierce SuperSignal ${ }^{\circledast}$ chemiluminescence kit (Pierce, Rockford, IL). The primary antibodies used were as follows: mouse anti-phospho-ERK (T202/Y204), rabbit anti-ERK, rabbit anti-Src, rabbit anti-PP2A C subunit, rabbit antiphospho-CREB (Ser133), and rabbit anti-CREB purchased from Cell Signaling Technology (Beverly, MA); rabbit anti-phospho-ER $\alpha$ (Ser118) and rabbit anti-ER $\alpha$ purchased from Santa Cruz Biotechnology (Santa Cruz, CA); rabbit anti-phospho- PP2A (Y307) purchased from Abcom Biotechnology (Cambridge, UK), rabbit antiphospho-Src (Tyr527) purchased from Sigma (St. Louis, $\mathrm{MO}) ; \beta$-actin purchased from Boster Biotechnology (WuHan, HB, China). Blots were then washed four times for $15 \mathrm{~min}$ and visualized using enhanced chemiluminescence (ECL; Amersham Biosciences, Piscataway, NJ).

\section{PP2A activity assay}

PP2A activity was measured with a molybdate dye-based phosphatase assay kit (Promega, Madison, WI). Assays were performed according to the manufacturer's protocol. Briefly, the samples were homogenized in lysis buffer (Tris-HCl 50, pH 7.4, EDTA 0.1, DTT 1, PMSF 0.1 mM, 1\% Triton X-100 and 1\% protease inhibitor cocktail) in ice water. Samples were filtered through Sephadex G-25 columns to remove free phosphate. The samples were then added to a reaction premix which contained phosphopeptide substrate, $5 \times$ PP2A reaction buffer $(250 \mathrm{mM}$ imidazole, pH 7.2, 1 mM EGTA, 0.1\% $\beta$-mercaptoethanol, and $0.5 \mathrm{mg} / \mathrm{ml} \mathrm{BSA}$ ) and storage buffer in 96-well plates. The reactions were terminated by adding a molybdate dye/ additive mixture and incubating at $37^{\circ} \mathrm{C}$ for $30 \mathrm{~min}$. Finally, phosphotase activity in the samples were measured using a spectrophotometer (Molecular Devices, Sunnyvale, CA) at $630 \mathrm{~nm}$ wavelength.

\section{Data analysis}

In each case, for semi-quantitative analysis, the data from at least four animals are expressed as the mean \pm standard deviation. Statistical analysis was conducted using an analysis of variance (ANOVA) followed by the NewmanKeuls test. Comparisons between two groups were performed using a t-test. $P$ values less than 0.05 were considered significant.

\section{Abbreviations}

ERK: Extracellular signal - regulated kinase; PP2A: Protein phosphotase 2A; CREB: cyclic AMP response elementbinding protein; ER $\alpha$ : estrogen receptor $\alpha$; MAPK: mitogen-activated protein kinase; NMDA: N-methyl-Daspartic acid; L-VGCC: L-type voltage-gated calcium channels; IP3: Inositol 1,4,5-Triphosphate; PTK: protein tyrosine kinase; SH: Src homology; SU: SU6656; Ct: Cantharidin; 4VO: four-vessel occlusion; i.c.v.: intracerebral ventricular; BSA: bovine serum albumin; PAGE: polyacrylamide gel electrophoresis; Veh: vehicle.

\section{Authors' contributions}

$\mathrm{XH}, \mathrm{XW}$ carried out the 4-VO model and sample preparation, participated in the Western blot analysis and drafted the manuscript. JX participated in PP2A activity assay. JZ participated in the I.c.v. infusion. XH, JG conceived of the study, and participated in its design and coordination. All authors read and approved the final manuscript.

\section{Acknowledgements}

The work was supported by grants from the National Natural Science Foundation of China (No. 3087 I200) and Medical Foundation of Department of Health of Jiangsu Province (No. H200749).

\section{References}

I. Starkov AA, Chinopoulos C, Fiskum G: Mitochondrial calcium and oxidative stress as mediators of ischemic brain injury. Cell Calcium 2004, 36:257-264.

2. Gao J, Duan B, Wang DG, Deng XH, Zhang GY, Xu L, Xu TL: Coupling between NMDA Receptor and Acid-Sensing Ion Channel Contributes to Ischemic Neuronal Death. Neuron 2005 48:635-646. 
3. Song B, Ma C, Gong S, Yuan Z, Li D, Liu W, Li W, Chen R, Zhu X, Zeng J, Han Y, Li M: Extracellular signal-regulated kinases are not involved in activity-dependent survival or apoptosis in cerebellar granule neurons. Neurosci 2006, 407:2/4-218.

4. Mehta SL, Manhas N, Raghubir R: Molecular targets in cerebral ischemia for developing novel therapeutics. Brain Res Rev 2007, 54:34-66.

5. Rusanescu G, Qi H, Thomas SM, Brugge JS, Halegoua S: Calcium influx induces neurite growth through a Src-Ras signaling cassette. Neuron 1995, 15:1415-1425.

6. Guo J, Wu HW, Hu G, Han X, De W, Sun YJ: Sustained activation of Src-family tyrosine kinases by ischemia: a potential mechanism mediating extracellular signal-regulated kinase cascades in hippocampal dentate gyrus. Neurosci 2006, | 43:827-836.

7. Be\&\#×0|42;towski J, Wójcicka G, Trzeciak J, Marciniak A: $\mathbf{H}_{\mathbf{2}} \mathbf{O}_{\mathbf{2}}$ and Src-dependent transactivation of the EGF receptor mediates the stimulatory effect of leptin on renal ERK and $\mathrm{Na}^{+}$, K+-ATPase. Peptides 2006, 27( ( 2):3234-3244.

8. Moelling K, Schad K, Bosse M, Zimmermann S, Schweneker M: Regulation of Raf-Akt Cross-talk. J Biol Chem 2002, 277(34):31099-106.

9. Van Kanegan MJ, Adams DG, Wadzinski BE, Strack S: Distinct protein phosphatase 2A heterotrimers modulate growth factor signaling to extracellular signal-regulated kinases and Akt. J Biol Chem 2005, 280:36029-36036.

10. Miglietta A, Bozzo F, Gabriel L, Bocca C, Canuto RA: Extracellular signal-regulated kinase $I / 2$ and protein phosphatase $2 A$ are involved in the antiproliferative activity of conjugated linoleic acid in MCF-7 cells. Br J Nutr 2006, 96:22-27.

II. Zhao J, Wu HW, Chen YJ, Tian HP, Li LX, Han X, Guo J: Protein phosphatase 2A-negative regulation of the protective signaling pathway of $\mathrm{Ca2}+/ \mathrm{CaM}$-dependent ERK activation in cerebral ischemia. J Neurosci Res 2008, 86( I 2):2733-45.

12. Mao L, Yang L, Arora A, Choe ES, Zhang G, Liu Z, Fibuch EE, Wang JQ: Role of Protein Phosphatase 2A in mGluR5-regulated MEK/ERK Phosphorylation in Neurons. J Biol Chem 2005, 280(13): $12602-12610$.

13. Chung H, Nairn AC, Murata K, Brautigan DL: Mutation of Tyr307 and Leu309 in the protein phosphatase 2A catalytic subunit favors association with the alpha 4 subunit which promotes dephosphorylation of elongation factor-2. Biochemistry 1999 38(32): $1037 \mid-10376$

14. Liu W, Akhand AA, Takeda K, Kawamoto Y, Itoigawa M, Kato M, Suzuki H, Ishikawa N, Nakashima I: Protein phosphatase 2Alinked and -unlinked caspase-dependent pathways for downregulation of Akt kinase triggered by 4-hydroxynonenal. Cell Death Differ 2003, I0(7):772-781.

15. Wu HW, Li HF, Guo J: Spry2-mediated inhibition of the Ras/ ERK pathway through interaction with Src kinase following cerebral ischemia. Brain Inj 2008, 22(3):275-28I.

16. Lee S, Yoon S, Kim DH: A high nuclear basal level of ERK2 phosphorylation contributes to the resistance of cisplatin-resistant human ovarian cancer cells. Gynecologic Oncology 2007, 104:338-344.

17. Xie W, Duan R, Safe S: Activation of adenosine deaminase in MCF-7 cells through IGF-estrogen receptor alpha crosstalk. J Mol Endocrinol 200I, 26:217-228.

18. Tang HY, Lin HY, Zhang S, Davis FB, Davis PJ: Thyroid hormone causes mitogen-activated protein kinase-dependent phosphorylation of the nuclear estrogen receptor. Endocrinology 2004, 1 45:3265-3272.

19. Glaros S, Atanaskova N, Zhao C, Skafar DF, Reddy KB: Activation Function-I Domain of Estrogen Receptor Regulates the Agonistic and Antagonistic Actions of Tamoxifen. Molecular Endocrinology 2005, 20:996-1008.

20. Zhao $X$, Lorenc $H$, Stephenson $H$, Wang $Y$ J, Witherspoon $D$, Katzenellenbogen B, Pfaff D, Vasudevan N: Thyroid hormone can increase estrogen-mediated transcription from a consensus estrogen response element in neuroblastoma cells. Proc Natl Acad Sci USA 2005, 102:4890-4895.

21. Park EM, Joh TH, Volpe BT, Chu CK, Song G, Cho SA: Neuroprotective role of extracellular signal regulated kinase in $N$ acetyl-O-methyldopamine-treated hippocampal neurons after exposure to in vivo ischemia. Neurosci 2004, I 23: I47-I 54.
22. D'Cruz BJ, Logue ES, Falke E, DeFranco DB, Callaway CW: Hypothermia and ERK activation after cardiac arrest. Brain Research 2005, 1064: I08-II 8.

23. Christen V, Treves S, Duong FH, Heim MH: Activation of endoplasmic reticulum stress response by hepatitis viruses upregulates protein phosphatase 2A. Hepatology 2007, 46(2):558-565.

24. Zhou XW, Gustafsson JA, Tanila H, Bjorkdahl C, Liu R, Winblad B, Pei J]: Tau hyperphosphorylation correlates with reduced methylation of protein phosphatase 2A. Neurobiol Dis 2008, $31(3): 386-94$

25. Li Z, Hosoi Y, Cai K, Tanno Y, Matsumoto Y, Enomoto A, Morita A Nakagawa K, Miyagawa K: Src tyrosine kinase inhibitor PP2 suppresses ERK I/2 activation and epidermal growth factor receptor transactivation by $\mathbf{X}$-irradiation. Biochem Biophys Res Commun 2006, 341 (2):363-368.

26. Choe ES, Parelkar NK, Kim JY, Cho HW, Kang HS, Mao L, Wang JQ: The protein phosphatase I/2A inhibitor okadaic acid increases C REB and Elk-I phosphorylation and c-fos expression in the rat striatum in vivo. I Neurochem 2004, 89(2):383-390.

27. Kim SH, Seo MS, Jeon WJ, Yu HS, Park HG, Jung GA, Lee HY, Kang UG, Kim YS: Haloperidol regulates the phosphorylation level of the MEK-ERK-p90RSK signal pathway via protein phosphatase $2 A$ in the rat frontal cortex. Int J Neuropsychopharmacol 2008, I I (4):509-5 I7.

28. Tan B, Long $X$, Nakshatri H, Nephew KP, Bigsby RM: Striatin-3 gamma inhibits estrogen receptor activity by recruiting a protein phosphatase. J Mol Endocrinol 2008, 40(5): 199-2I0.

29. Chen J, Martin BL, Brautigan DL: Regulation of protein serinethreonine phosphatase type-2A by tyrosine phosphorylation. Science 1992, 257(5074): 126|-4.

30. Zhu H, Yang $H$, Owen MR: Combined microarray analysis uncovers self-renewal related signaling in mouse embryonic stem cells. Syst Synth Biol 2007, I(4): I7I-8I.

3I. Thandi S, Blank JL, Challiss RA: Group-I metabotropic glutamate receptors, $\mathrm{mGlu} \mathrm{I}$ a and $\mathrm{mGlu5a}$, couple to extracellular signal-regulated kinase (ERK) activation via distinct, but overlapping, signalling pathways. J Neurochem 2002, 83: I I39-53.

32. Miñano A, Xifró X, Pérez V, Barneda-Zahonero B, Saura CA, Rodríguez-Alvarez J: Estradiol facilitates neurite maintenance by a Src/Ras/ERK signalling pathway. Mol Cell Neurosci 2008, 39(2): |43-5|

33. Hu BR, Liu CL, Park DJ: Alteration of MAP kinase pathways after transient forebrain ischemia. I Cereb Blood Flow Metab 2000, 20:1089-1095.

\section{Publish with Bio Med Central and every scientist can read your work free of charge}

"BioMed Central will be the most significant development for disseminating the results of biomedical research in our lifetime."

Sir Paul Nurse, Cancer Research UK

Your research papers will be:

- available free of charge to the entire biomedical community

- peer reviewed and published immediately upon acceptance

- cited in PubMed and archived on PubMed Central

- yours - you keep the copyright
BioMedcentral 